\title{
Perfil dos casos de síndrome respiratória aguda grave obtidos por um laboratório de referência em saúde pública
}

\author{
Profile severe acute respiratory syndrome cases obtained by a public health reference laboratory \\ Perfil de casos de síndrome respiratório agudo grave obtenidos por un laboratório de referencia de \\ salud pública
}

\begin{abstract}
Resumo
Objetivo: Analisar o perfil dos casos de SRAG notificados e resultados obtidos por um Laboratório de Referência em Saúde Pública. Metodologia: Trata-se de um estudo de tipo transversal, desenvolvido a partir das fichas de notificação e resultados notificados de SRAG obtidos através do Núcleo de Vigilância do LACEN-GO. Utilizou-se o software Epi info 3.5.4 para transcrição dos resultados, análise das variáveis contidas na ficha de notificação e teste de Qui-quadrado (x2). Resultados: Foram analisadas 691 fichas de notificação de SRAG e seus respectivos resultados notificados. A idade média dos casos foi de 33 anos, a principal amostra biológica foi a secreção de oro e nasofaringe e para a identificação da etiologia viral utilizou a RT-PCR. Quanto ao perfil epidemiológico a maioria dos casos eram do sexo feminino, não gestantes, escolaridade maior que 8 anos, não vacinados e houve necessidade do uso de antiviral. Os principais sinais e sintomas foram tosse, febre e desconforto respiratório. A principal comorbidade foi doença cardiovascular crônica. Houve a necessidade de hospitalização, o principal padrão radiológico encontrado foi o infiltrado intersticial e ocorreu o uso de unidade de terapia intensiva. O principal agente etiológico viral diagnosticado foi o vírus Influenza A/H1N1pdm09. Conclusão: A vigilância epidemiológica da Influenza através da Notificação de SRAG, incluindo a vigilância de SG em unidades sentinelas é uma importante ferramenta para a saúde pública e se tornou o fator fundamental para a prevenção, contenção, vigilância e manejo terapêutico de pacientes.
\end{abstract}

Palavras-chave: Influenza; SRAG; Vigilância Laboratorial.

\section{Abstract}

Objective: To analyze the profile of notified SARS cases and results obtained by a Public Health Reference Laboratory. Methodology: This is a cross-sectional study, developed from the notification forms and notified results of SRAG obtained through the LACEN-GO Surveillance Nucleus. The Epi info 3.5.4 software was used to transcribe the results, analyze the variables contained in the notification form and the Chi-square test (x2). Results: 691 SRAG notification forms and their reported results were analyzed. The average age of the cases was 33 years, the main biological sample was the secretion of the oro and nasopharynx and for the identification of the viral etiology, the RT-PCR was used. Regarding the epidemiological profile, most of the cases were female, non- 
pregnant, had an education higher than 8 years, were not vaccinated and there was a need for the use of antiviral drugs. The main signs and symptoms were cough, fever and respiratory distress. The main comorbidity was chronic cardiovascular disease. There was a need for hospitalization, the main radiological pattern found was the interstitial infiltrate and the intensive care unit was used. The main viral etiologic agent diagnosed was the Influenza A / H1N1pdm09 virus. Conclusion: The epidemiological surveillance of Influenza through SARS Notification, including surveillance of SG in sentinel units, is an important tool for public health and has become the fundamental factor for the prevention, containment, surveillance and therapeutic management of patients.

Keywords: Influenza; SRAG; Laboratory Surveillance.

\section{Resumen}

Objetivo: Analizar el perfil de casos notificados de SARS y los resultados obtenidos por un Laboratorio de Referencia de Salud Pública. Metodología: Se trata de un estudio transversal, desarrollado a partir de los formularios de notificación y resultados notificados del SRAG obtenidos a través del Núcleo de Vigilancia LACEN-GO. Se utilizó el software Epi info 3.5.4 para transcribir los resultados, analizar las variables contenidas en el formulario de notificación y la prueba de Chi-cuadrado (x2). Resultados: Se analizaron 691 formularios de notificación del SRAG y sus resultados reportados. La edad promedio de los casos fue de 33 años, la principal muestra biológica fue la secreción de la oro y nasofaringe y para la identificación de la etiología viral se utilizó la RT-PCR. En cuanto al perfil epidemiológico, la mayoría de los casos fueron mujeres, no embarazadas, con estudios superiores a los 8 años, no vacunadas y hubo necesidad del uso de medicamentos antivirales. Los principales signos y síntomas fueron tos, fiebre y dificultad respiratoria. La principal comorbilidad fue la enfermedad cardiovascular crónica. Hubo necesidad de hospitalización, el principal patrón radiológico encontrado fue el infiltrado intersticial y el uso de una unidad de cuidados intensivos. El principal agente etiológico viral diagnosticado fue el virus Influenza A / H1N1pdm09. Conclusión: La vigilancia epidemiológica de la Influenza a través de la Notificación del SARS, incluida la vigilancia de SG en unidades centinela, es una herramienta importante para la salud pública y se ha convertido en el factor fundamental para la prevención, contención, vigilancia y manejo terapéutico de los pacientes.

Palabras-clave: Influenza, SRAG, Vigilancia de Laboratorio.

\section{Introdução}

As infecções do trato respiratório representam importante causa de morbidade e mortalidade em todo o mundo, responsáveis por mais de quatro milhões de óbitos anualmente. Apesar da importância de bactérias como agentes de infecções do trato respiratório, vírus são responsáveis por cerca de 80\% destas infecções (Falsey et al., 2014; Who, 2014).

A Síndrome Respiratória Aguda Grave (SRAG) é caracterizada como agravo de um quadro de Síndrome Gripal (SG) ou gripe, onde o indivíduo de qualquer idade apresente os seguintes sinais e sintomas: febre alta, tosse, dispneia, mialgia, dor de garganta, saturação $\mathrm{O}_{2}<95 \%$ e desconforto respiratório, podendo evoluir rapidamente o óbito (MS, 2015). O vírus influenza é um dos principais vírus respiratórios que levam ao desenvolvimento de SRAG. Existem três tipos de Influenza Sazonal: A, B, C. O vírus influenza A e B apresentam maior importância clínica, devido a estimativa que $75 \%$ das infecções são ocasionadas pela cepa do tipo A e as demais pelo B, sendo a $\mathrm{C}$ raramente leva ao desenvolvimento de casos graves, apenas infecções brandas. Os subtipos mais comuns em circulação são o Influenza A (H1N1pdm09) e o A(H3N2), sendo o subtipo H1N1pdm09 que ocasionou a pandemia em 2009 (Bresee, 2018). Os vírus influenza sofrem frequentes mutações e possuem elevada transmissibilidade e distribuição global, com fácil disseminação em epidemias sazonais e podem gerar pandemias. $\mathrm{O}$ principal mecanismo de transmissão ocorre por meio de partículas eliminadas do trato respiratório do indivíduo contaminado ao tossir, falar, espirrar e pelas mãos (Who, 2018). É muito elevada em ambientes domiciliares, creches, escolas, ambientes fechados e em meios de transporte como aviões, navios, transportes coletivos, etc, dependendo do número e da intensidade dos contatos entre as pessoas de diferentes faixas etárias (Bresee, 2018).

Segundo a Organização Mundial da Saúde, a ocorrência de casos da influenza pode levar a hospitalização e a morte, ocorrem principalmente entre os grupos de alto risco como em idosos, crianças e entre portadores de comorbidades. No cenário mundial estima-se que epidemias por influenza resultem em cerca de 3 a 5 milhões de casos de doença grave e cerca de 290.000 a 650.000 mortes (Who, 2018). No Brasil é obrigatória a notificação dos casos de SRAG, através do Sistema de Vigilância Epidemiológica da Influenza, incluindo a vigilância de SG em unidades sentinelas, para o monitoramento dos vírus respiratórios circulantes, e além de permitir o monitoramento da demanda de atendimentos por SG (MS, 2015). Em virtude do 
exposto, o presente estudo teve como objetivo analisar o perfil dos casos de SRAG notificados e resultados obtidos em um Laboratório de Referência em Saúde Pública.

\section{Metodologia}

Trata-se de um estudo de tipo transversal, desenvolvido a partir das fichas de notificação e resultados notificados de SRAG obtidos através do Núcleo de Vigilância Laboratorial do Laboratório Central de Saúde Pública Dr. Giovanni Cysneiros (LACEN-GO), no período de 2015 a 2018.

Foram analisadas 691 fichas de notificação de SRAG e seus respectivos resultados notificados do diagnóstico etiológico viral realizados pelo LACEN-GO. Foram considerados como critério de inclusão os casos confirmados de SRAG através dos resultados notificados pelo Núcleo de Vigilância Laboratorial do LACEN-GO, no período de estudo. Foram excluídos da análise, dados das fichas de notificação que não estavam preenchidas adequadamente, no qual impossibilitava a verificação da possível associação entre os dados clínicos, laboratoriais e epidemiológicos.

Utilizou-se o software Epi info 3.5.4 (Centers for Disease Control and Prevention - CDC, Atlanta) para transcrição dos resultados e análise das variáveis contidas na ficha de notificação compulsória. As variáveis inclusas foram: sexo, gestação, escolaridade, vacinação prévia, sinais e sintomas, diagnóstico etiológico, hospitalização, raio x de tórax, uso de suporte ventilatório, uso de unidade de terapia intensiva, tipo de amostra coletada e principais comorbidades. Realizou-se o teste de Qui-quadrado $\left(x^{2}\right)$ para verificar uma possível associação entre as faixas etárias dos casos de SRAG com a vacinação prévia e entre a prescrição de Fosfato de Oseltamivir de acordo com os sinais e sintomas, sendo $p<0,005$ considerado estatisticamente significativo com intervalo de confiança de $95 \%$. Os resultados foram descritos através forma absoluta e frequência. $\quad O$ presente estudo foi aprovado pelo Comitê de Ética do Hospital de Doenças Tropicais Dr. Anuar Auad (HDT) e pelo Laboratório Central de Saúde Pública Dr. Giovanni Cysneiros (LACEN-GO) através do número de parecer 2.621.411 em 23 de março de 2018.

\section{Resultados}

Foram analisadas 691 fichas de notificação de SRAG e seus respectivos resultados notificados pelo Núcleo de Vigilância Laboratorial do LACEN-GO. A idade média dos casos notificados foi de 33 anos. A maior frequência agrupada entre idades foram entre idosos ( $\geq 60$ anos) em 103 (15,1\%) casos, seguido de 41-50 anos em 99 (14,3\%) casos e 0-1 ano em $90(13,0 \%)$ casos. A maioria das amostras biológicas coletadas para o diagnóstico etiológico viral foi através da secreção de oro e nasofaringe em $687(99,4 \%)$ casos e os demais $4(0,6 \%)$ utilizou-se como amostra o tecido post-mortem. Para a identificação da etiologia viral utilizou-se em 100\% das amostras biológicas a Reação em Cadeia da Polimerase Transcriptase Reversa (RT-PCR). Quanto ao perfil epidemiológico a maioria dos casos eram do sexo feminino, não gestantes, escolaridade maior que 8 anos, não vacinados e houve necessidade do uso de antiviral (Fosfato de Oseltamivir), descritos na tabela 1. 
Tabela 1 - Perfil Epidemiológico dos Casos de Síndrome Respiratória Aguda Grave obtidos pelo Laboratório Central de Saúde Pública de Goiás.

\begin{tabular}{lcc}
\hline Variável & Casos (n) & Frequência (\%) \\
\hline Sexo & 394 & 57,0 \\
Feminino & 297 & 43,0 \\
Masculino & & \\
& & \\
Gestação & 48 & 12,2 \\
Sim & 346 & 87,8 \\
Não & &
\end{tabular}

\section{Escolaridade}

$\begin{array}{lcc}<4 \text { anos } & 1 & 0,1 \\ 4-8 \text { anos } & 181 & 26,2 \\ >8 \text { anos } & 346 & 50,1 \\ \text { Não informado } & - & - \\ \text { Ignorado } & 163 & 23,6\end{array}$

\section{Vacinação Prévia}

Sim $138 \quad 20,0$

Não $\quad 550 \quad 79,6$

Não informado

$3 \quad 0,4$

\section{Uso de Antiviral}

\begin{tabular}{lcc} 
Sim & 542 & 78,6 \\
Não & 145 & 20,9 \\
Não informado & 4 & 0,5 \\
\hline
\end{tabular}

Fonte: Autores.

Os principais sinais e sintomas descritos com maior frequência foram tosse, febre, desconforto respiratório, dispneia, saturação <95\%, mialgia, dor de garganta (Tabela 2). As principais comorbidades descritas foram doença cardiovascular crônica em 8,2\% casos, pneumopatias crônicas em 6,4\%, obesidade em 4,8\% e diabetes mellitus em $2,7 \%$ dos casos. 
Tabela 2. Sinais e Sintomas dos apresentados pelos casos de Síndrome Respiratória Aguda Grave.

\begin{tabular}{lcc}
\hline Variável & Casos & Frequência (\%) \\
\hline Tosse & 678 & 98,1 \\
Febre & 677 & 98,0 \\
Desconforto Respiratório & 669 & 97,8 \\
Dispneia & 600 & 86,8 \\
Saturação $<95 \%$ & 515 & 74,5 \\
Mialgia & 355 & 51,4 \\
Dor de Garganta & 286 & 41,4 \\
Cefaleia & 58 & 8,3 \\
Dor Torácica & 25 & 3,7 \\
Diarreia & 22 & 3,2 \\
Vômitos & 8 & 1,2 \\
\hline
\end{tabular}

Fonte: Autores.

A necessidade de hospitalização foi descrita em $643(93,1 \%)$ casos. O principal padrão radiológico encontrado foi o infiltrado intersticial em 388 (56,2\%) casos, seguido por radiografia normal 117 (16,9\%), misto 40 (5,7\%), consolidação 41 $(6 \%)$ e não foram realizados raio X em 105 (15,2\%) casos. A necessidade em utilizar unidade de terapia intensiva ocorreu em $184(26,6 \%)$ casos e 507 (73,4\%) não ocorreu. Não houve a necessidade de utilização de suporte ventilatório em 401 (57,4\%) casos, seguido do uso de suporte ventilatório não invasivo em $180(26,0 \%)$ e a utilização do suporte invasivo ocorreu em 114 $(16,5 \%)$ casos. Observou-se que a maioria dos casos em relação as faixas etárias agrupadas, não possuíam vacinação prévia contra H1N1. Ao verificar possível associação estatística entre as faixas etárias dos casos de acordo com a vacinação prévia, não houve relevância estatística ( $p>0,005)$. O uso de antiviral (Fosfato de Oseltamivir) foi utilizado em 542 (78,6\%) casos. Ao verificar associação estatística entre a prescrição de Fosfato de Oseltamivir de acordo com os sinais e sintomas descritos pelos casos, salientamos que não houve relevância estatística $(p>0,005)$.

Os principais agentes etiológicos virais diagnosticados foram: Influenza A/H1N1pdm09 em 380 (54,9\%) casos, seguido de Influenza B em 91 (13,2\%), Influenza A/H3N2 em 88 (12,7\%), os demais vírus respiratórios são descritos na tabela 3. 
Tabela 3. Agentes Etiológicos causadores de Síndrome Respiratória Aguda Grave.

\begin{tabular}{lcc}
\hline Etiologia Viral & Casos & Frequência (\%) \\
\hline Influenza A/H1N1pdm09 & 380 & 54,9 \\
Influenza B & 91 & 13,2 \\
Influenza A/H3N2 & 88 & 12,7 \\
Vírus Sincicial & 64 & 9,3 \\
Respiratório & 28 & 4,1 \\
Metapneumovírus & 20 & 2,9 \\
Adenovírus & 14 & 2,0 \\
Parainfluenza 3 & 2 & 0,3 \\
Parainfluenza 1 & 4 & 0,6 \\
Não Detectável & & \\
\hline
\end{tabular}

Fonte: Autores.

\section{Discussão}

Neste estudo os casos notificados e os resultados do diagnóstico etiológico de SRAG no período de 2015 a 2017 , observamos que a média da idade foi de 33 anos, com maior frequência em idosos ( $\geq 60$ anos), seguido de 41-50 anos e 0-1 anos. Cavalieri et al. (2016) em um estudo em Santa Catarina, encontrou uma média de idade de 31 anos, semelhante ao encontrado em nosso estudo. Porém Ng et al. (2016) descreve que em relação as faixas etárias a maior positividade de testes confirmatórios, são maiores entre crianças e adolescentes por volta de 10 a 15 anos, além de serem importantes fontes de transmissão devido à idade escolar. As crianças com idade de 1 a 5 anos são as principais fontes de transmissão dos vírus entre suas famílias e na comunidade. Estima-se que uma pessoa infectada seja capaz de transmitir o vírus para até dois contatos não imunes (Plans-Rubió, 2012). Cowling et al. (2013) em estudo realizado na China, observou-se dados que corroboram os achados deste estudo em relação ao predomínio de casos na faixa de 60 anos.

A maioria das amostras biológicas coletadas para o diagnóstico, serem da secreção de oro e nasofaringe e o método de diagnóstico utilizado ser a Reação em Cadeia da Polimerase - Transcriptase Reversa (RT-PCR) que é considerado o método sensível, específico e versátil para identificação de vírus respiratórios, estão de acordo com métodos que o Ministério da Saúde preconiza para diagnóstico viral de vírus respiratórios (MS, 2017).

$\mathrm{Na}$ análise relacionada ao sexo dos casos, foi evidenciado um predomínio do sexo feminino (57\%), isto vai ao encontro de dados das vigilâncias epidemiológicas no Brasil e na Europa, onde variam de 55,6\% (Lenzi et al., 2011; SchuelterTrevisol et al., 2012; Lan et al., 2013) e 57\% (Fiebig et al., 2011). Na categoria gestante observou-se $48(12,2 \%)$ casos, o que corrobora com o estudo de Cavalieri et al. (2016). Entretanto Maraví-Poma et al. (2011) demonstrou que a gravidez estava significativamente associada a quadros de pneumonia viral, decorrente do vírus Influenza. O risco de complicações é muito alto, principalmente no terceiro trimestre de gestação, mantendo-se elevado no primeiro mês após o parto, reforçando a necessidade de vacinação (Paddock et al., 2012; Bresse, 2018)

Em relação à escolaridade, ou seja, o tempo de estudo, observou-se que a maioria dos casos possuíam mais de 8 anos de estudo. Porém Lan et al. (2013) observou que quanto mais baixo o nível de escolaridade, maior a proporção de notificação, no qual existe uma relação entre mortalidade e analfabetismo ou desconhecimento do nível de escolaridade. 
A vacinação prévia contra H1N1 encontrada no presente estudo, é visto que 79,6\% dos casos não eram vacinados previamente. A vacinação contra influenza mostra-se como uma das medidas mais efetivas para a prevenção da influenza grave e de suas complicações (Fiore et al., 2007; Bresse, 2018). Existem diversas vacinas contra a influenza que diferem quanto à sua composição e que podem ter diferentes indicações, de acordo com faixa etária (Paddock et al., 2012; Bricks et al., 2014). Em populações não vacinadas, a maioria das mortes por influenza sazonal é registrada em idosos. Alguns estudos demonstram que a vacinação pode reduzir entre $32 \%$ a $45 \%$ o número de hospitalizações por pneumonias, de $39 \%$ a $75 \%$ a mortalidade global e 50\% nas doenças relacionadas à influenza (Fiore et al., 2007; Bresee, 2018). As políticas de vacinação contra influenza são de suma importância, observando-se que a vacinação embora não confira proteção completa, previne aproximadamente $30 \%$ dos casos fatais e não fatais em idosos, independentemente da etiologia (Michels et al., 2011).

A necessidade do uso de antiviral (Fosfato de Oseltamivir) foi constatado em 78,6\% dos casos, não corroborando com Cavalieri et al. (2016) no qual encontrou em apenas 6,8\% dos casos. Em ensaios em adultos, o uso de antiviral diminuiu o tempo para o primeiro alívio dos sintomas por 16,8 horas (Jefferson et al., 2016). Em todos os casos, o início do tratamento deve ser preferencialmente nas primeiras 48 horas após o início dos sintomas (MS, 2015)

Os principais sinais e sintomas encontrados como tosse, febre, desconforto respiratório, dispneia, saturação <95\%, mialgia, dor de garganta estão de acordo com a literatura pesquisada (Fiore et al., 2007; Bricks et al., 2014; Bresee, 2018). As principais comorbidades relatadas foram a doença cardiovascular crônica em 8,2\% dos casos, seguida de pneumopatias crônicas em 6,4\%, obesidade em 4,8\% e diabetes mellitus em 2,7\% casos. Esses resultados discordam dos resultados encontrados por Cavalieri et al. (2016) onde encontrou-se frequência de obesidade em 37,4\% dos casos, seguida de doença pulmonar obstrutiva crônica em $20 \%$, diabetes mellitus em $15,2 \%$ e doença hematológica em $12.5 \%$ dos casos.

O vírus influenza causa exacerbação de comorbidades. É importante ressaltar que as frequências de outras comorbidades como doenças cardíacas, metabólicas e neoplásicas aumentam com a idade, e que existe associação que pacientes com doenças crônicas muitas vezes não são vacinados por não estarem cientes de sua condição de risco ou por falta de recomendação médica (Coloqhuoun et al., 1997; Merts et al., 2013).

Não possível identificar associação estatística entre as faixas etárias dos casos de acordo com a vacinação prévia, porém Mazon et al. (2016) observou relevância estatística entre as idades e a vacinação prévia. Também não foi possível identificar associação estatística entre o uso de antiviral (Fosfato de Oseltamivir) e os sinais e sintomas, porém Mazon et al.(2016) observou relevância nos sintomas como dificuldade respiratória $(p=0,005)$, mialgia $(\mathrm{p}=0,003)$ e cansaço $(p=0,016)$.

Os principais agentes etiológicos virais descritos foram os vírus Influenza A/H1N1pdm09 em 380 (54,9\%) casos, seguido de Influenza B em 91 (13,2\%), Influenza A/H3N2 em 88 (12,7\%), corroborando com estudos encontrados. É interessante ressaltar que no Brasil e em outros países, o vírus influenza A/H1N1pdm09 tem acometido principalmente adultos (40-60 anos), o vírus influenza B em crianças, adolescentes e adultos jovens e o vírus influenza A/H3N2 possui maior impacto em idosos. Entretanto, todas os subtipos podem causar infecções graves e levar a mortes em pessoas de qualquer faixa etária (Allard et al., 2012; Freitas, 2013; Freitas et al., 2013; Nitsch-Osuch et al. 2013; Jayasundara et al., 2014).

\section{Conclusão}

A vigilância epidemiológica da Influenza através da Notificação de SRAG, incluindo a vigilância de $\mathrm{SG}$ em unidades sentinelas é uma importante ferramenta para a saúde pública e se tornou o fator fundamental para a prevenção, contenção, vigilância e manejo terapêutico de pacientes.

$\mathrm{O}$ fato das amostras positivas para Influenza A/H1N1pdm09, Influenza B, Influenza A/H3N2 serem provenientes de unidades hospitalares, sugere-se uma associação destes subtipos a quadros mais graves da doença, podendo levar os pacientes 
rapidamente ao óbito. Os resultados observados sinalizam para a necessidade de um acompanhamento longitudinal para melhor esclarecer a dinâmica de transmissão do vírus em longo prazo e aprimorar as estratégias de controle.

\section{Referências}

Allard, R. et al. (2012). Invasive bacterial infections following influenza: a time-series analysis in Montréal, Canada, 1996- 2008. Oxford. 6(4), 268-275.

Breese, J. S.; Fry, A. M.; Sambara, S.; Cox, N. J. (2018). Inactivated Influenza Vaccines. In: Plotkin, S.; Orenstein, W.; Offit, P.; Eduwards, K. M. Plotkin`s Vaccines. Philadelphia: Saunders Elsevier, 456-488.

Bricks, L. F. et al. (2014). Influenza em crianças o que há de novo? Journal of Health and Biological Sciences. 3(2), $125-134$.

Cavalieri, G. C. et al. (2016). Perfil Epidemiológico dos Casos de Influenza A em Santa Catarina, Brasil no ano de 2012. Arq. Catarin Med. 45(2),79-90.

Cowling, B. J. et al. (2013). Comparative epidemiology ofhuman infections with avian Influenza A (H7N9) and A(H5N1) viruses in China. Lancet. 382(9887), $129-137$.

Coloquhoun, A. J. et al. (1997). Effectiveness of influenza vaccine in reducing hospital admissions in people with diabetes. Epidemiology and Infection. 119(3), 335-341.

Falsey, A. R. et al. (2014). Respiratory syncytial virus and other respiratory viral infections in older adults with moderate to severe influenza-like illness. The Journal Infectious Disease. 209(2), 1873-1881.

Fiebig, L. et al. (2011). Avian Influenza A (H5N1) in humans: new insights from a line list of World Health Organization confirmed cases. Euro Surveill. $16(32), 1-10$

Fiore, A. E. et al. (2015). Prevention and control of influenza. Recommendations of the Advisory Committee on Immunization Practices (ACIP), 2007. Morbidity and Mortality Weekly Report. 56, 1-24.

Freitas, F. T. et al. (2014). Reitas, F. T. Sentinel surveillance of influenza and other respiratory viruses, Brazil, 2000-2010. The Brazilian Journal of Infectious Disease. 17(1), 62-68.

Freitas, A. R. et al. (2013). Mortality associated with influenza in tropics, state of São Paulo, Brazil, from 2002 to 2011 : the pre-pandemic, pandemic, and post-pandemic periods. Influenza Research and Treatment. 4(1), 20-28.

Jayasundara, K. et al. (2016). Natural attack rate of influenza in unvaccinated children and adults: a meta-regression analysis. BMC Infectious Disease. 1(2).

Jefferson, T. et al. (2014). Oseltamivir for influenza in adults and children: systematic review of clinical study reports and summary of regulatory comments. BM. 314 .

Lan, Y. et al. (2013). Epidemiology of pandemic Influenza A/H1N1 virus during 2009-2010 in Taiwan. Virus Res. 117(2), 46-54.

Lenzi, L. et al. (2011). Study of the relationship between sociodemographic characteristics and new Influenza A (H1N1). Braz J Infect Dis. 15(5), 457-467.

Maraví-Poma, E. et al. (2011). Severe 2009 H1N1 Influenza in pregnant women in Spain. Crit Care Med. 39(1), 945-951.

Mazon, L. M. et al. (2016). Perfil epidemiológico de pacientes com síndrome gripal e síndrome respiratória aguda grave. Sáude em Revista. 2(4).

Michels, B. et al. (2011). A systematic review of the evidence on the effectiveness and risks of inactivated influenza vaccines in different target groups. Vaccine. 29(49), 9159-9170.

Ministério da Saúde. (2015). Secretaria de Vigilância em Saúde. Departamento de Vigilância das Doenças Transmissíveis. Protocolo de tratamento de Influenza, 1(1), 1-41.

Ministério da Saúde. (2015). Protocolo de Manejo Clínico de Síndrome Respiratória Aguda Grave-SRAG. Brasília, DF.

Ministério da Saúde. (2017). Guia de vigilância em saúde. Volume único.

Mertz, D. et al. (2013). Populations at risk for severe or complicated influenza illness: systematic review and meta-analysis. British Medical Journal. 347(3).

$\mathrm{Ng}$, S. et al. (2016). The timeline of influenza virus shedding in children and adults in a household transmission study of influenza in Managua, Nicaragua. The Pediatric Infectious Disease Journal. 35(5), 582-586.

Nitsch-Ouch, A. et al. (2013). Clinical features and outcomes of influenza A and B infections in children. Advances in Experimental Medicine and Biology. $788(2), 89-96$.

Paddock, C. D. et al. (2012). Myocardial injury and bacterial pneumonia contribute to the pathogenesis of fatal influenza B virus infection. The Journal of Infectious Disease. 205(6), 895-905.

Plans-rubió, P. The vaccination coverage required to establish herd immunity against influenza viruses. Preventive Medicine, v.55, n.1, p.72-77, 2012.

Schuelter-Trevisol, F. et al. (2012). Perfil epidemiológico dos casos de gripe A na região sul de Santa Catarina, Brasil, na pidemia de 2009. Rev Panam Salud Publica. 32(1), 82-86. 
Research, Society and Development, v. 10, n. 6, e51710616056, 2021

(CC BY 4.0) | ISSN 2525-3409 | DOI: http://dx.doi.org/10.33448/rsd-v10i6.16056

World Health Organization. (2018). Infection prevention and control of epidemic- and pandemic- prone acute respiratory infections in health care. Geneva: Word Health Organization, 2014a. http://www.who.int/mediacentre/factsheets/fs141/en/.

World Health Organization. (2018). Influenza (seasonal). Fact sheet. 2018; http://www.who.int/mediacentre/factsheets/fs211/en) 